\title{
Interfacial flow computations using adaptive Eulerian-Lagrangian method for spacecraft applications
}

\author{
Jaeheon Sim and Wei Shyy*,† \\ Department of Aerospace Engineering, University of Michigan, Ann Arbor, MI 48109, U.S.A.
}

\begin{abstract}
SUMMARY
Understanding the interfacial dynamics and fluid physics associated with the operation of spacecraft is important for scientific as well as engineering purposes. To help address the issues associated with moving boundaries, interfacial dynamics, and spatial-temporal variations in time and length scales, a 3-D adaptive Eulerian-Lagrangian method is extended and further developed. The stationary (Eulerian) Cartesian grid is adopted to resolve the fluid flow, and the marker-based triangulated moving (Lagrangian) surface meshes are utilized to treat the phase boundary. The key concepts and numerical procedures, along with the selected interfacial flow problems are presented. Specifically, the liquid fuel draining dynamics in different flow regimes, and the liquid surface stability under vertically oscillating gravitational acceleration are investigated. Direct assessment of experimental measurement and scaling analysis is made to highlight the computational performance of the present approach as well as the key fluid physics influenced by the given flow parameters. Copyright (c) 2010 John Wiley \& Sons, Ltd.
\end{abstract}

Received 27 April 2010; Revised 14 August 2010; Accepted 27 September 2010

KEY WORDS: multiphase flow; interfacial dynamics; Eulerian-Lagrangian method; adaptive grid; space craft fuel draining; thrust oscillation; Faraday waves

\section{INTRODUCTION}

When designing and operating a spacecraft, management of the propellants and other fluid supply is one of the major concerns. The unoriented draining and sloshing motion under microgravity have a huge influence on the vehicle dynamics due to the shift in its center of mass. Furthermore, the variation on the amount of fuel delivered to the combustion chamber may result in a failure of the engine operation and consequently in a mission failure.

Contrary to the behavior of fluid on the ground, the fuel draining process under microgravity condition can cause unexpected large interface distortion and slosh waves, resulting in fast vapor ingestion and large liquid residuals. Petrash et al. studied experimentally the effect of weightlessness on the liquid-vapor interface in a spherical tank without draining [1]. Various drop tower tests on the draining process under microgravity followed and they found that the shape of the tank bottom and the position of the outlet have little influence on the draining process, while the pressuring gas diffuser and outlet baffle minimize the interface distortion [2,3]. Typically, the Froude number, the ratio of inertia to gravitational forces, is correlated with draining phenomena under normal gravity [4]. However, the Froude number has diminished influence on the draining process under microgravity; instead, the Weber number, which measures the ratio of inertia to surface tension

${ }^{*}$ Correspondence to: Wei Shyy, Department of Aerospace Engineering, University of Michigan, Ann Arbor, MI 48109, U.S.A.

†E-mail: weishyy@umich.edu 
forces, is found to have stronger influence on the draining procedure in weightlessness $[3,4]$. Symons defined a draining parameter by grouping the Weber number and Froude number to cover phenomena ranging from micro to normal gravity conditions, and categorized three different draining flow regimes according to the flow characteristics of the draining process. [5].

Thrust oscillation is also crucial in rocket designs since it has influence on the whole rocket system including astronauts' safety, and might even result in a critical structural failure of the rocket. Active controls have been applied to reduce such a dangerous pogo oscillation and shock absorbers have been installed for the safety of astronauts and payloads by mitigating thrust oscillation [6]. However, more detailed study on the cause and influence of thrust oscillation is still required since it is related to engine pressure and fuel delivery system such as fuel/oxidizer pump and injectors. Moreover, the bubble formations due to thrust oscillation may cause vapor ingestion into combustor and subsequent engine failure. The enhanced heat transfer from droplets causes ullage pressure rise and has huge influence on the fuel tank structure. When we focus on the surface wave stability due to thrust oscillation, it has a very similar mechanism to the well-known Faraday wave, a parametrically excited surface wave. In 1831, Faraday found that parametric vertical vibration generates subharmonic standing waves on the fluid surface [7]. Various surface wave modes and patterns, and the influence of tank geometries are studied [8-10]. Perinet et al. investigated numerically the spatio-temporal spectrum of both square and hexagonal patterns in a rectangular parallelepiped container [11]. A liquid depth to radius ratio is also an important parameter due to its influence on the non-linear resonance. The standing surface wave becomes non-linear and unstable at certain specific conditions, and critical standing wave height and exciting frequency/magnitude level are proposed as a criteria for surface instability [12]. Goodridge investigated experimentally the surface wave stability for fluids with different viscosities by measuring the exciting acceleration level where the surface becomes unstable, namely the threshold acceleration, in a cylindrical container [13-15]. The threshold acceleration depends on surface tension and viscosity as well as forcing frequency.

Considering microgravity conditions in a typical spacecraft environment, the capillary effect becomes very important due to small Bond number, the ratio of body (or gravitational) forces to surface tension forces. Thus, a study on the multiphase flow including interfacial dynamics is required in order to understand the liquid fuel dynamics in a spacecraft. However, experimental studies are limited because the microgravity conditions are hard to realize on the ground. Drop tower tests and in-flight tests have been conducted, but their short operational times prohibited simulating practical engineering problems. Thus, a high-fidelity numerical simulation of such a multiphase flow in a space environment is crucial to compensate the limitation of experiment.

The multiphase flow includes an interface between different phases having distinct physical properties, and consequently involves identifying interface location and modeling interfacial dynamics, including a steep jump in fluid properties in response to surface tension effects. Various methods have been proposed and improved and each method has its own relative strengths and weaknesses $[16,17]$.

In order to represent interface in moving boundary computations, three main categories exist as reviewed by Shyy et al. [16]; Lagrangian (moving grid), Eulerian (stationary grid), and EulerianLagrangian methods. The Lagrangian method uses body-fitted grid for tracking interfaces, and is accurate since interfacial conditions can be applied at the exact interface location. Both structured grid [18] and unstructured grid [19] have been implemented. However, maintaining grid quality is difficult, and computationally expensive to use when large deformation or movements occur. The Eulerian (stationary grid) method extracts the interface location via post processing with a help of a scalar function on a stationary computational grid, and its simplicity and natural handling of topological change makes these methods very popular. The level-set (LS) method [20] and the volume of fluids (VOF) method [21] are well-known Eulerian method. A main difference between these two methods is the choice of the scalar function; a distance function is used in the LS method whereas the volume fraction in the VOF. However, implicit interface extraction causes errors in interface location or surface tension computation. The Eulerian-Lagrangian method utilizes a separate set of moving (Lagrangian) mesh and associated marker/tag system representing 
interface on a stationary (Eulerian) grid used to compute the flow fields. Thus, the interface can be tracked explicitly, and these methods possess several desirable features when compared with the Lagrangian or Eulerian method. The immersed boundary method (IBM) [22-25] and the sharp interface method $[16,26-28]$ are in this category.

Another challenging issue is handling of the interfacial dynamics even if we know the location and shape of the interface at a given time. Across an interface, in general, the pressure and viscous stresses show discontinuities and fluid properties suddenly jump due to surface tension. Moreover, phase change includes mass and heat transfer across an interface. How to treat such a phase discontinuity and associated capillary effects can be classified into two categories: the sharp interface method and the continuous interface method (or diffuse-interface method). In the sharp interface method, the jump condition across phase boundary is satisfied exactly on the interface of zero-thickness. Compared with the sharp interface method, the continuous interface method smoothes out the fluid properties and interfacial forces around phase boundary instead of a zero-thickness interface. A popular and highly successful continuous interface technique in the Eulerian-Lagrangian method, proposed by Peskin, is the IBM [22]. It utilizes a single fluid formulation for the entire domain by smoothing out the fluid property and modeling the surface tension forces as a momentum source term within finite range. The continuous interface method is implemented successfully by many researchers to simulate large-deformable or merger/breakup fluid flows $[29,30]$. The sharp interface method can be adopted in the Lagrangian method having body-fitted grid since the interface is aligned with grid, and the interfacial dynamics can be applied exactly on the interface [18]. On the other hand, Shyy et al. [16] and Ye et al. [27,31] presented a sharp interface approach based on the Eulerian-Lagrangian approach, which treats the interfacial physics without smearing. Mittal et al. [32] further implemented a sharp interface method with the help of ghost cells by reconstructing the solution on those to satisfy the jump condition across interface. The cut-cell [27,33] and ghost fluid methods [34] belong to this category. Usually, a fluid/solid interface is modeled by a sharp interface method due to small deformation and there is no need to solve solid phase while fluid/fluid interface is modeled by a continuous method because it is easier to implement and has better capability handling various multiphase problems even with large deformation and moving interfaces.

In this paper, we have further developed a 3-D adaptive Eulerian-Lagrangian method previously reported in References [30,35,36]. Both continuous and sharp interface methods are implemented in a unified framework using the contact line force model with the local slip condition for complex moving multiphase flow computations having both fluid and solid interfaces. This numerical method utilizes the stationary (Eulerian) frame to resolve the flow field, and the marker-based triangulated moving (Lagrangian) surface meshes to treat the phase boundary interfaces. The large-deformable fluid boundaries are modeled using a continuous interface method, and the surface tension between fluid interfaces is smeared within finite distance. The solid boundaries are treated by a sharp interface method along with the ghost cell method by reconstructing the solution on the ghost cell based on the known solid boundary condition. The contact line where the fluid interface meets the solid boundary is modeled using a contact line force model, which enforces the given contact angle dynamically. For moving contact line treatment, local slip condition is applied around the contact line. The adaptive Cartesian grid method is implemented to resolve the sufficient computation resolution, especially around interface with effective computation. The liquid fuel draining process under microgravity and the stability of the liquid-gas interface dynamics due to vertically oscillating gravitational acceleration of varying frequency and amplitudes are investigated via direct numerical simulations with experimental validation.

\section{NUMERICAL METHODS}

In the present study, a marker-based 3-D adaptive Eulerian-Lagrangian method is implemented to perform incompressible interfacial flow computations around irregular solid geometries. The bulk flow variables are solved on a stationary (Eulerian) background grid, whereas interface variables 


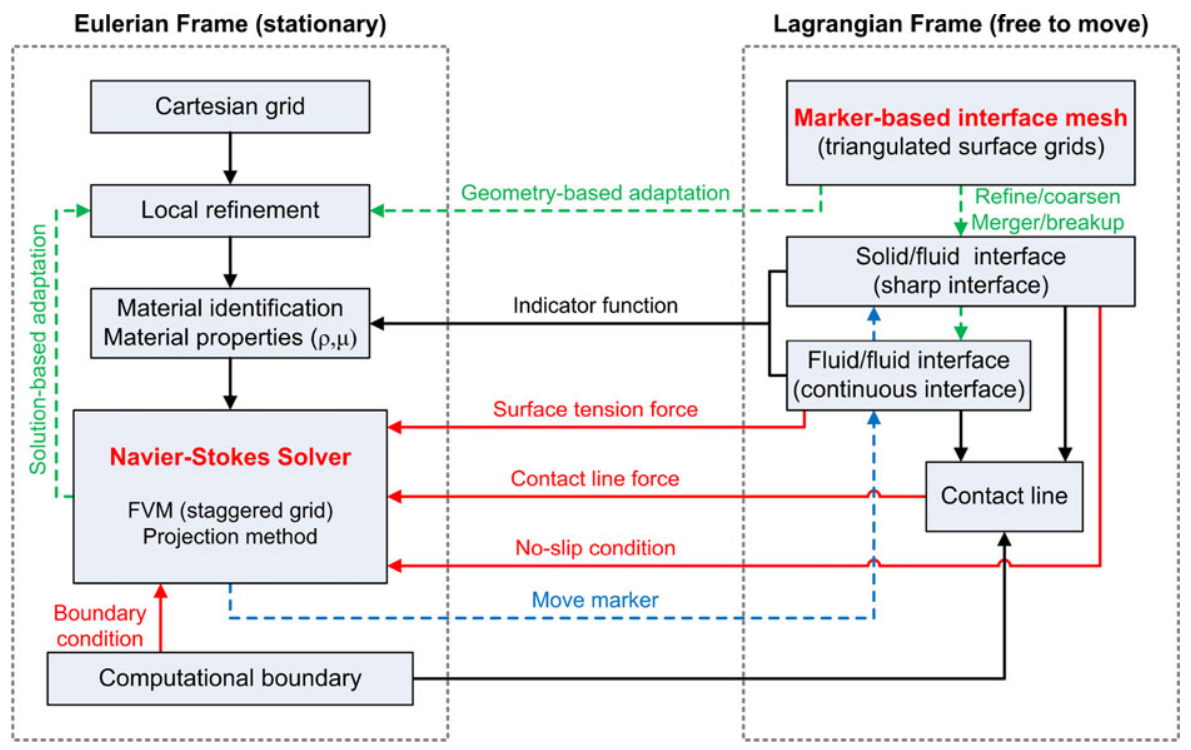

Figure 1. Summary of the present adaptive Eulerian-Lagrangian method.

are handled by moving (Lagrangian) surface meshes. Both continuous and sharp interface methods are implemented in a unified framework with the help of the contact line force model having the local slip condition. The components of the numerical algorithm and their interactions are summarized in Figure 1, and a detailed numerical method can be found in the previous work $[30,35,36]$.

\subsection{Governing equations}

Incompressible Navier-Stokes equations for mass and momentum conservation are given in Equations (1) and (2), respectively, which account for the interfacial dynamics due to surface tension effects of fluid interfaces as a forcing term $\left(\mathbf{F}_{\mathrm{f}}\right)$. Here, $\mathbf{V}$ is the velocity vector and $\rho, \mu$, and $p$ are the density, viscosity, and pressure, respectively.

$$
\begin{gathered}
\nabla \cdot \mathbf{V}=0 \\
\frac{\partial(\rho \mathbf{V})}{\partial t}+\nabla \cdot(\rho \mathbf{V} \mathbf{V})=-\nabla p+\frac{1}{R e} \nabla \cdot \mu\left(\nabla \mathbf{V}+\nabla^{\mathrm{T}} \mathbf{V}\right)+\frac{1}{F r} \rho \mathbf{g}+\frac{1}{W e} \mathbf{F}_{\mathrm{f}}
\end{gathered}
$$

Here, all variables are non-dimensionalized by a characteristic velocity $\left(\mathbf{V}_{\text {ref }}\right)$, length scale $\left(L_{\text {ref }}\right)$, standard gravity $\left(\mathbf{g}_{0}\right)$, liquid material properties (density $\rho_{1}$ and viscosity $\mu_{1}$ ), and surface tension $(\sigma)$ between phases. The non-dimensional parameters of the Reynolds $(R e)$, Froude $(F r)$, and Weber ( $W e$ ) numbers in Equation (2) are defined as Equations (3)-(5).

$$
\begin{gathered}
R e=\frac{\rho_{1} \mathbf{V}_{\text {ref }} L_{\text {ref }}}{\mu_{\mathrm{l}}} \\
F r=\frac{\mathbf{V}_{\text {ref }}^{2}}{\mathbf{g}_{0} L_{\text {ref }}} \\
W e=\frac{\rho_{\mathrm{l}} \mathbf{V}_{\text {ref }}^{2} L_{\text {ref }}}{\sigma}
\end{gathered}
$$

In addition, the capillary number $(\mathrm{Ca})$ and bond number $(\mathrm{Bo})$ can be defined as $C a=W e / R e=$ $\left(\mu_{\mathrm{l}} \mathbf{V}_{\text {ref }}\right) / \sigma$ and $B o=W e / F r=\left(\rho_{1} g_{0} L_{\text {ref }}^{2}\right) / \sigma$, respectively.

A single set of equations formulation for all fluid phases in the whole domain is made possible by smoothing out the discontinuous material properties across the interface. This is achieved with 
the help of an approximate Dirac-delta function $\left(\delta_{h}\right)$ and an indicator function $(I)$. The approximate Dirac-delta function, originally proposed by Peskin [22], is implemented over finite thickness of four cell width instead of the analytical form of the Dirac-delta function, which has a non-zero value only at the zero-thickness interface. The indicator function is a scalar function varying from zero to one smoothly across the interface and has a value of 0.5 at the interface location. The smoothed fluid properties, such as density and viscosity, are computed using Equations (6) and (7).

$$
\begin{gathered}
\rho=\rho_{2}+\left(\rho_{1}-\rho_{2}\right) I \\
\frac{\rho}{\mu}=\left(\frac{\rho}{\mu}\right)_{2}+\left[\left(\frac{\rho}{\mu}\right)_{1}-\left(\frac{\rho}{\mu}\right)_{2}\right] I
\end{gathered}
$$

Separate indicator functions are designated for fluid and solid interfaces, respectively. The solid interface is modeled by a sharp interface method with zero-thickness, and the fluid interface is modeled by a continuous interface method with diffused finite thickness. The indicator function is computed using a discrete form of the Heaviside step function in Equation (8) by integrating the 1-D form of the discrete Dirac-delta function. This approach is known as being applicable more generally than a Poisson equation solver method since it requires only distance information from interface, and thus gives accurate values even at the boundaries [35].

$$
\boldsymbol{I}(\mathbf{x})=\mathbf{H}(r=\mathbf{n} \cdot(\mathbf{x}-\mathbf{X}))=\int_{-\infty}^{r} \delta_{h}(h) \mathrm{d} h
$$

The incompressible Navier-Stokes equations (1) and (2) are solved using a projection method, where an intermediate velocity field is computed and projected into a divergence-free space. The convection term is discretized using the third-order ENO scheme in space and the second-order Runge-Kutta integration in time. The central difference scheme along with the Crank-Nicholson method is implemented for the viscous term. The pressure Poisson equation for the velocity correction is solved using the conjugate gradient method. The finite volume method is implemented with the staggered grid arrangement, where the face-normal velocity components are stored on the face centers of Cartesian grid cells and other variables (pressure, density, viscosity, etc.) are stored at the cell centers.

\subsection{Interface representation and tracking}

In this Eulerian-Lagrangian method, the interface is represented by surface meshes composed of massless markers and triangular elements with connectivity information. The corresponding data structure is established via line-segments in 2-D and triangles in 3-D domains, as represented in Figure 2(a). Markers store the surrounding elements' indices, whereas elements store the neighboring elements based on the edge that they share. For boundary elements, negative values are used to refer a computational boundary or to intersect solid elements as shown in Figure 2(b).
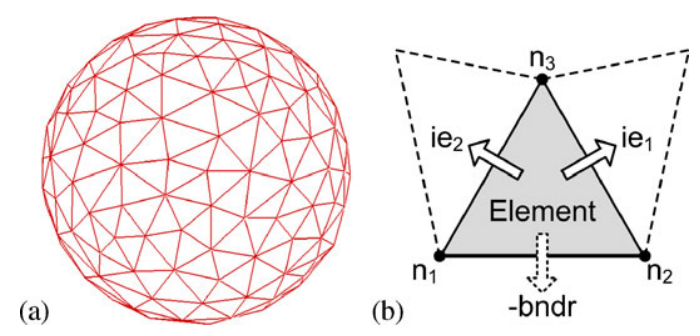

Figure 2. Interface representation: (a) Interface surface represented by massless markers and triangular elements and (b) connectivity information for the boundary triangular element with two inner neighboring elements. 
The marker locations, denoted by $\mathbf{X}$ in the Lagrangian frame, are updated from the velocities at its location, $\mathbf{V}(\mathbf{X})$, in Equation (9).

$$
\frac{\partial \mathbf{X}}{\partial t}=\mathbf{V}(\mathbf{X})
$$

Fluid interfaces use the computed flow field on the Eulerian grid to obtain the marker velocities as shown in Equation (10), where the discrete Dirac-delta function, $\delta_{h}(\mathbf{x}-\mathbf{X})$, is employed for converting the Eulerian velocity field, $\mathbf{V}(\mathbf{x})$, to the Lagrangian form, $\mathbf{V}(\mathbf{X})$. On the other hand, solid interfaces use the prescribed velocity field to advance the marker points.

$$
\mathbf{V}(\mathbf{X})=\int_{V} \mathbf{V}(\mathbf{x}) \delta_{h}(\mathbf{x}-\mathbf{X}) \mathrm{d} V
$$

As the marker points advance to a new position in time, the interface surface mesh can exhibit an uneven distribution of marker points. In order to maintain consistent computational accuracy, the spacing between marker points is rearranged by adding or deleting markers whenever two markers come too close or are too distant from each other. The criteria for the distance between the adjacent markers are estimated based on the requirements posed by the background grid and the function relating Eulerian quantities to Lagrangian quantities. For fluid interfaces, the length of the edge between neighboring markers are maintained from $\Delta / 3$ to $\Delta$, where $\Delta$ is the Eulerian grid spacing, for continuous representation of transferred quantities by the Dirac-delta function in Equation (10). A less demanding criterion can be used for solid interfaces since it deforms less and does not require transfer between Lagrangian and Eulerian quantities. The volume errors during these processes can be recovered by adjusting the location of the modified marker in its normal direction, as discussed by Singh and Shyy [30].

The level-contour-based interface reconstruction technique with connectivity information is implemented to handle topological changes such as a merger or break-up. With connectivity information, the time-consuming reconstruction is facilitated only in case of a merger/break-up by examining the possibility of topological changes at prescribed time intervals. These reconstruction procedures can be done locally for a specific interface body, and the errors in phase volume after reconstruction are explicitly corrected by perturbing the markers in local normal direction.

\subsection{Fluid interface treatment: continuous interface method}

The continuous interface method is implemented for all fluid phases by a single fluid formulation due to its effectiveness in modeling highly deformable fluid interfaces. The surface force defined in Equation (11), where $\sigma$ is the surface tension and $\kappa$ is the curvature of interface, is added as a source term in momentum equation (2).

$$
\mathbf{F}_{\mathrm{f}}=\int_{A} \sigma \kappa \delta_{h}(\mathbf{x}-\mathbf{X}) \mathrm{d} A
$$

The surface force of the interface is also transformed from the Lagrangian quantity $(\mathbf{X})$ to the Eulerian quantity ( $\mathbf{x})$ via the approximate discrete Dirac-delta function, $\delta_{h}(\mathbf{x}-\mathbf{X})$. The computation on a discretized interface element (line-segments in 2-D and triangles in 3-D) is conducted by a line integral form shown in Equation (12) with normal (n) and tangent vectors (t) of elements instead of a direct curvature computation since the direct curvature computation using interpolation-based methods is numerically sensitive and often requires some form of data smoothing, and does not enforce surface tension conservation on a closed surface.

$$
\delta \mathbf{f}=\int_{\text {element }} \sigma(\mathbf{n} \times \nabla) \times \mathbf{n} \mathrm{d} A=\sum_{i=\text { edges }} \sigma(\mathbf{t} \otimes \mathbf{n})_{i} \Delta s
$$

\subsection{Solid interface treatment: sharp interface method}

Solid interface is modeled using the sharp interface method by reconstructing solution fields around interfaces to incorporate the given boundary condition of solid surface. Solution reconstruction can 


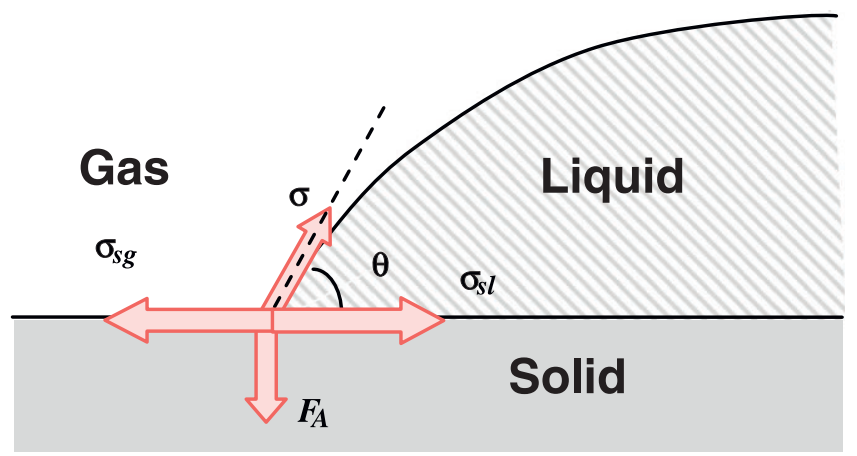

Figure 3. Forces at tri-junction (contact line). $F_{A}$ represents the adhesive forces.

be conducted either on the fluid side or solid side. Both approaches work well for a single phase flow around solid surfaces. However, the fluid velocity reconstruction method tends to ignore the influence of surface tension and moving phase boundary by reconstructing fluid velocities directly. Alternatively, the velocity reconstruction on the solid side can be implemented via ghost cells, which are defined as solid cells having at least one neighboring fluid cell. This approach works well even with a moving contact line where fluid interface meets solid interface.

The solution reconstruction on the ghost cell is conducted by a linear or bi/trilinear interpolation scheme using solutions at the solid surface and at the known fluid cells. Avoiding vague extrapolation due to the location of ghost cell in solid phase, the interpolation can be conducted first at an imaginary point in fluid phase, and then, extrapolated to the ghost cells based on the known value at a solid surface.

\subsection{Contact line treatment}

When we consider a fluid-fluid interface intersecting a solid surface, the treatment of the trijunction locations, called a contact line, is required to account for the interactions of all three phases, fluid-fluid-solid. One of the most discussed issues for modeling these contact lines with Navier-Stokes equations is that the imposed no-slip condition leads to a non-integrable singularity in stress. In the present study, the contact line force is imposed with local slip condition to overcome this singularity issue.

In Figure 3, the angle at the contact line can be used for representing the balance of forces resulting from intermolecular forces between solid, liquid and gas phases. The tangential component of the resulting force at the contact line $\left(\mathbf{F}_{\mathrm{R}}\right)$ is shown in Equation (13). In static equilibrium, Equation (13) leads to the well-known Young-Laplace equation as given in Equation (14).

$$
\begin{gathered}
\mathbf{F}_{\mathrm{R}}=\sigma_{\mathrm{sg}}-\sigma_{\mathrm{sl}}-\sigma \cos \theta \\
\sigma_{\mathrm{sg}}=\sigma_{\mathrm{sl}}+\sigma \cos \theta_{0}
\end{gathered}
$$

In Equation (13), $\sigma_{\mathrm{sg}}$ is the surface force due to the interaction of solid and gas, $\sigma_{\mathrm{sl}}$ is the surface force due to the interaction of solid and liquid and $\sigma$ is the surface tension defined between liquid and gas. The given static contact angle is represented by $\theta_{0}$, and $\theta$ is the present contact angle at an instant. In this approach, the force at the contact line is obtained by plugging Equation (14) into Equation (13). This contact line force in Equation (15) accelerates or decelerates flow fields, and makes the present contact angle $(\theta)$ approach the prescribed static contact angle $\left(\theta_{0}\right)$ asymptotically.

$$
\mathbf{F}_{\mathrm{R}}=\sigma\left(\cos \theta_{0}-\cos \theta\right)
$$

The contact line force can be implemented with surface tension computation by modifying Equation (12) for open boundary edges, where the element connects to either a computational 


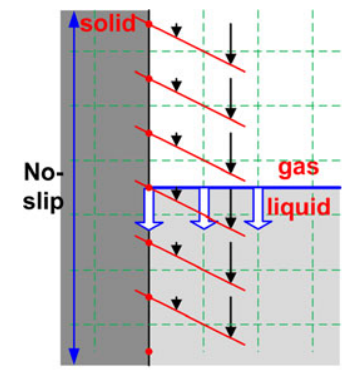

(a)

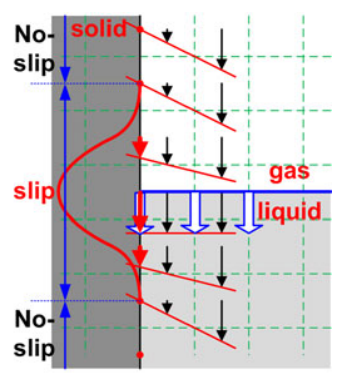

(b)

Figure 4. The comparison of velocity fields between no-slip and local slip condition: (a) No-slip condition with singularity and (b) local slip condition.

boundary or a solid interface. In Equation (16), the surface tension force from the interface curvature is applied to closed edges, and the contact line force is applied to open boundary edges.

$$
\boldsymbol{\delta} \mathbf{f}=\sum_{\substack{i=\text { closed } \\ \text { edges }}} \sigma(\mathbf{t} \otimes \mathbf{n})_{i} \Delta s+\sum_{\substack{i=\text { open } \\ \text { edges }}} \sigma\left(\cos \theta_{0}-\cos \theta\right)_{i} \mathbf{t} \Delta s
$$

Local slip condition is applied around the contact line on the solid boundary to simulate the moving contact line problems with better accuracy. The present model and solid boundary treatment work well with no-slip condition for steady and/or slowly moving fluid interface problems. However, the no-slip condition imposed on the solid leads to a non-integrable singularity in stress and retarded interface movement due to the discontinuous velocity fields in Figure 4(a). This singularity can be solved by applying the slip condition within finite distance from the contact line using the approximate Dirac-delta function in Figure 4(b). A perfect slip is applied exactly on the contact line, and partial slip is applied within two-cell distance as the continuous fluid interface is diffused.

\subsection{Adaptive grid}

Multiphase flow problems involve multiple length scales. In order to effectively resolve the flow features in such cases, an adaptive grid based on isotropic refinement is implemented. The cells are split into four and eight equal sibling cells in 2-D and 3-D, respectively, to better handle regions that require higher resolution. The grid is represented using unstructured data that connects cells through cell faces. The details of the algorithm can be found in Singh and Shyy [30]. Adaptation is performed based on the flow solution quality as well as the interface location for effective computation.

The geometry-based adaptation around interfaces allows better resolution in the near-wall region and phase boundary. Near-fluid interfaces, for which discontinuous flow properties smear across two layers of cells, refinement is performed to improve the resolution there to capture detailed flow structures more accurately. This fully resolved region around a fluid interface is further extended to six layers of cells to handle both the discrete Dirac function as well as a possibility for large deformation of the interface. On the other hand, solid interfaces, where interfacial dynamics are not smeared, triggers full refinement with two-cell layers from the wall.

Figure 5 illustrates the process of geometry-based adaptation starting from a uniform base grid and refining up to four levels around both fluid and solid interfaces. Furthermore, the computational cells are dynamically refined based on the solution of the flow field. The present implementation uses a curl-based adaptation criterion comparing the cell value with a standard deviation of the whole domain. 


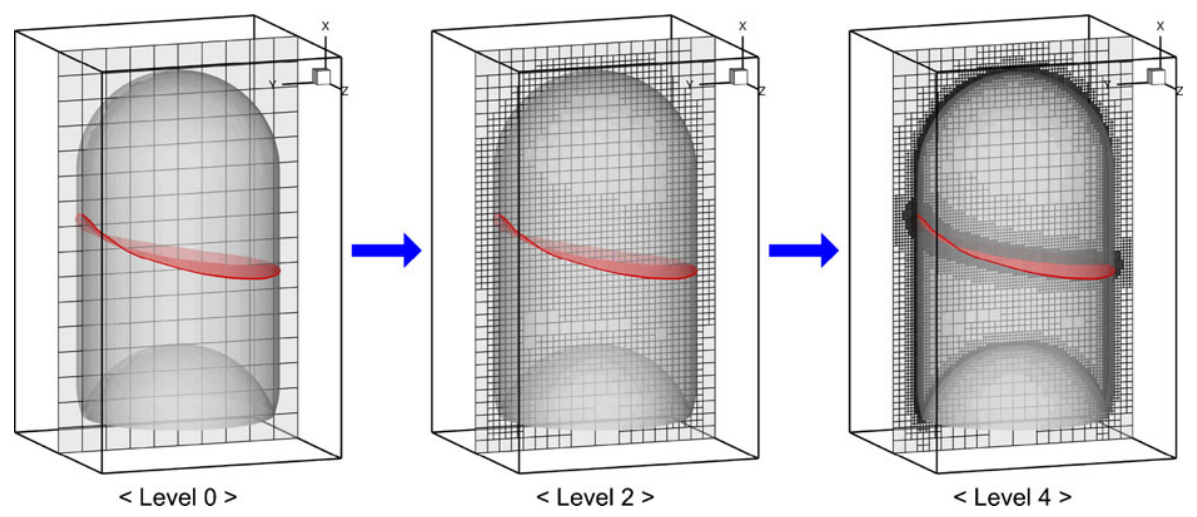

Figure 5. Snapshots of geometry-based grid adaptation. Grids are refined around fluid and solid interfaces of a concave-bottomed spacecraft fuel tank. Different adaptation levels from 0 to 4 are illustrated.

\section{RESULTS AND DISCUSSION}

\subsection{Fuel draining dynamics in a spacecraft}

The dynamics of the fuel delivery under microgravity are studied numerically and compared with an experimental study conducted by Symons at the zero-gravity facility of the NASA Lewis research center [5]. Figure 6 shows the experimental and numerical configuration. The hemispherical bottomed tank with a small draining hole is represented by a solid interface treatment on the stationary Cartesian grid. In the experiment, the inlet baffle (or pressurizing gas diffuser) of $\frac{1}{2}$ tank radius is installed to prevent direct impingement of the pressurizing gas onto the liquid fuel surface. It is known that the inlet baffle minimizes the interface distortion [2]. This equipment is modeled numerically by a mixed wall and an outlet boundary condition at the height of the inlet baffle. The non-dimensional initial liquid height, based on tank radius $(R)$, is set to 2 or 3 .

In Symons' experimental study, the draining phenomenon was measured after some transition time from normal gravity $\left(g_{0}\right)$ to the required microgravity $\left(0.015 g_{0}\right)$ condition with an equilibrium interface surface. The simulation is started from the end of the transition time, and it uses the initial equilibrium ellipsoidal interface shape. The specified mass flow rate is used except in one case, which is used for a more accurate comparison with the experimental data. As is done in the experiments, trichlorotrifluoroehhane is utilized as a substitute for liquid fuel, and air is employed as a pressurizing gas. The material properties used at $20^{\circ} \mathrm{C}$ are given in Table I.

The non-dimensional parameters, the Weber number $(W e)$, the Bond number $(B o)$, and the draining parameter $(\lambda)$, are defined with non-dimensional time $\left(t^{*}\right)$ to compare the results with the experiment and to distinguish the flow regime under microgravity in Equations (17)-(20).

$$
\begin{gathered}
W e=\frac{\rho_{1} V_{\mathrm{ref}}^{2} L_{\mathrm{ref}}}{\sigma}=\frac{\rho_{1} Q^{2}}{\pi^{2} \sigma R^{3}} \\
B o=\frac{W e}{F r}=\frac{\rho_{1} g R^{2}}{\sigma} \\
\lambda=\frac{W e}{B o+1} \\
t^{*}=\frac{t Q}{\pi R^{3}}
\end{gathered}
$$

where $t$ is dimensional time, $Q$ is the volume flow rate of the fuel draining, and $R$ is the characteristic length of the fuel tank, which is taken as the radius of tank for cylindrical geometry in this study as presented in Figure 6. 

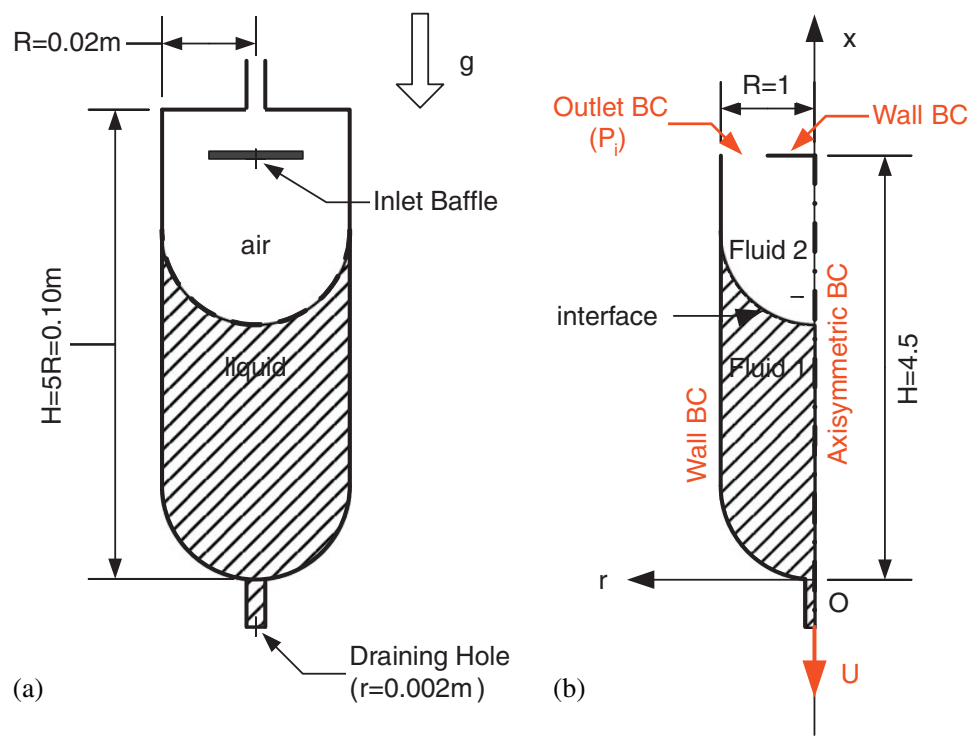

Figure 6. Geometry configuration: (a) Experiment and (b) present numerical study.

Table I. Material properties at $20^{\circ} \mathrm{C}$.

\begin{tabular}{lcc}
\hline Material properties at $20^{\circ} \mathrm{C}$ & Trichlorotrifluoroehhane (liquid) & Air (gas) \\
\hline Density $\left(\mathrm{kg} / \mathrm{m}^{3}\right)$ & $1.58 \times 10^{3}$ & 1.205 \\
Viscosity $(\mathrm{kg} / \mathrm{m} \cdot \mathrm{s})$ & $0.70 \times 10^{-3}$ & $1.821 \times 10^{-5}$ \\
Surface tension $(\mathrm{N} / \mathrm{m})$ & 0.0186 & \\
\hline
\end{tabular}

According to Symons, the flow characteristics of the draining process can be classified into three main categories: inertia-dominated, transition, and capillary-dominated regimes. In the present study, the Bond number is fixed at 5 with $g=0.015 g_{0}$, and various Weber numbers are chosen from 0.1 to 80 for characterizing each regime in terms of sloshing waves and residual volume. They correspond to draining parameters 0.01667 to 13.33 .

Direct simulation is conducted for a transition regime case, corresponding to the draining parameter $\lambda=0.16$, for which the experimental results are available. It should be noted that Symons quantified the draining parameter based on the mass flow rate measured under normal gravity [5]. However, the measured flow rate will be acceptable only if the pressurizing air pressure is much higher than the hydrostatic pressure of the liquid. It occurs in the inertia-dominated regime, where large flow rates require high air pressurizing. The difference of hydrostatic pressure between normal and micro gravity conditions cannot be ignored in capillary-dominated or transition regimes with small air pressurizing; it can cause errors in the measured flow rate. In order to establish an exact comparison basis, a procedure similar to the experiment is conducted by measuring the air pressure numerically for a given mass flow rate under normal gravity. Then, the obtained air pressure is implemented in a fuel draining simulation under microgravity. In this preliminary test, the reported draining parameter 0.18 in the experiment practically corresponds to 0.16 with $10 \%$ errors due to hydrostatic pressure differences. Thus, the simulation with draining parameter of 0.16 is compared with experimental data with draining parameter of 0.18 in Figure 7, where the non-dimensional height variation at the centerline and at the tank wall is shown. Contrary to the draining phenomenon under normal gravity, the fuel surface goes down with different velocities at the centerline and wall with varying downward speeds. This results in a jellyfish-like sloshing motion that repeats flat and elongated surfaces. The present study shows very consistent agreement with Symons' experimental study [5]. The sloshing motion and sudden vapor ingestion phenomena 


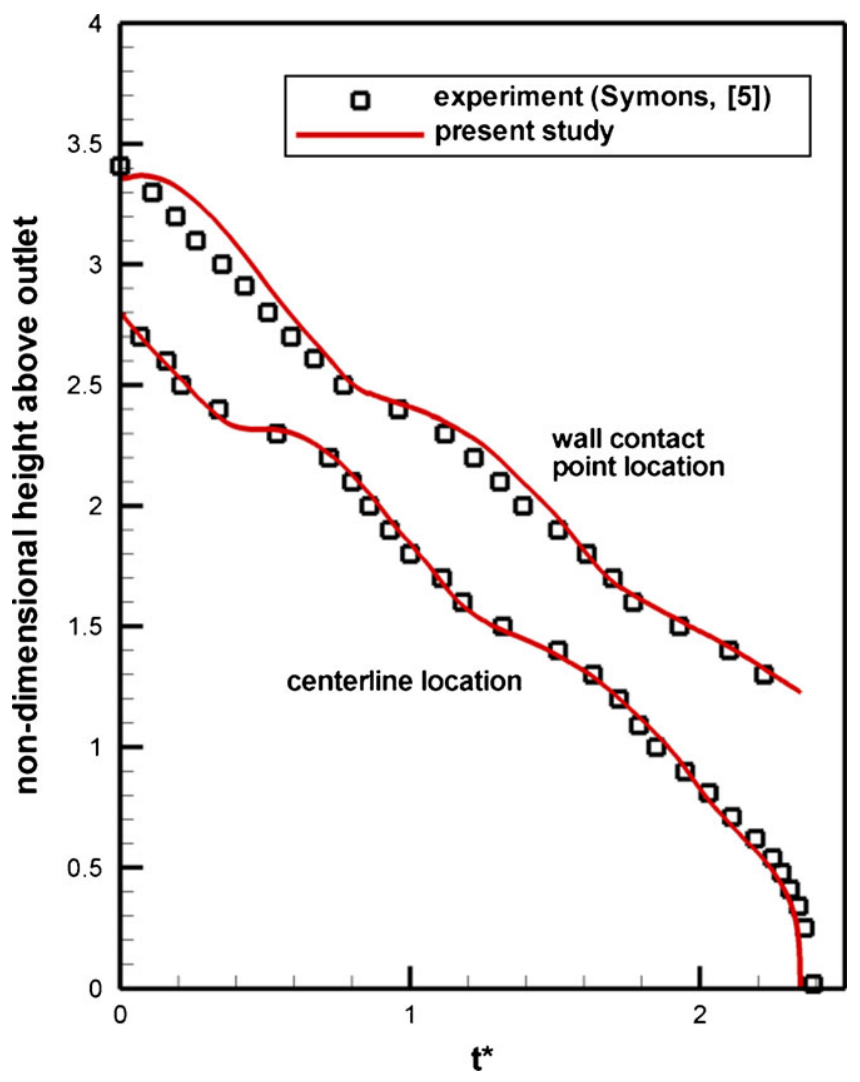

Figure 7. The comparison of present simulation results with experimental data by Symons.

are captured in detail, whereas the wall contact point location is slightly different in the beginning of the draining process, possibly as a result of differences in the initial conditions.

Under normal gravity, the liquid fuel in a tank goes down and maintains a flat interface shape during the draining process. Thus, the fuel can be used efficiently with little left over. However, the liquid fuel interface shows a very large distortion under microgravity conditions, since the small gravitational force does not flatten the liquid surface. Rather, interfacial force determines the fuel surface shape because it is competing against gravitational force. In this condition, the amount of usable fuel depends on the interface surface shape. Residual volume is defined as the remaining liquid volume in a tank at vapor ingestion, and thus, it informs us how much fuel can be used at the given condition. Figure 8 shows the non-dimensional residual volume $V_{\mathrm{r}}^{*}$ normalized by the hemispherical bottom volume for two different initial fill levels; 2 tank radii and 3 tank radii. The residual volume increases with the draining parameter until it reaches a certain value. At that point, it becomes insensitive to any changes in the draining parameter. With intermediate draining parameters, the residual volume shows oscillations due to the influence of sloshing waves. If a slosh wave is at its highest point at the incipience of vapor ingestion, the vapor ingestion is postponed, and this results in a decreased residual volume. The phase of the slosh waves determines the time of vapor ingestion, which in turn determines the remaining liquid residual volume. Thus, a higher initial fill level does not guarantee longer engine operation since vapor ingestion can occur earlier than with a lower initial fill level. The existence of three regimes can also be shown: a linear part with a small draining parameter, an oscillation part in the middle range, and the flat residual volume part with a high draining parameter.

The time history of the non-dimensional height at the centerline and at the wall attachment point is shown in Figure 9 for the mentioned regime conditions. A capillary-dominated regime is characterized by many slosh waves with small magnitudes. In this regime, the fluid level decreases 


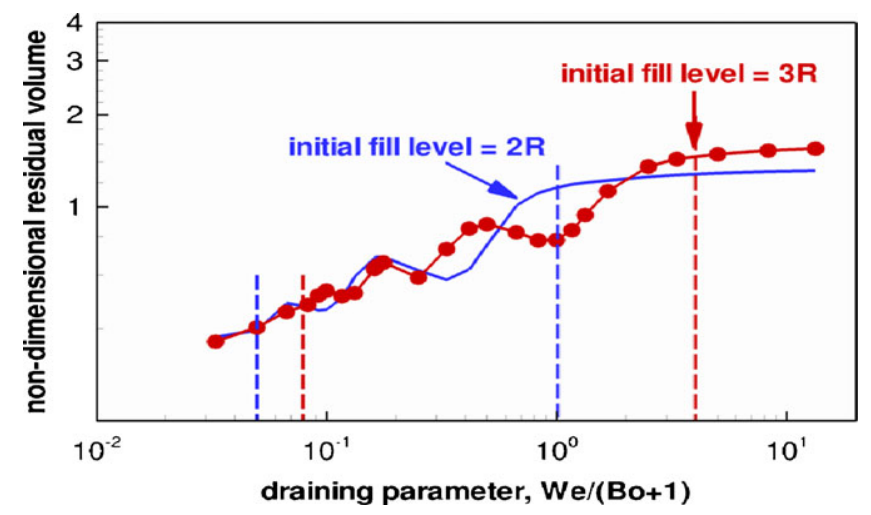

Figure 8. Non-dimensional residual volume in draining parameter.

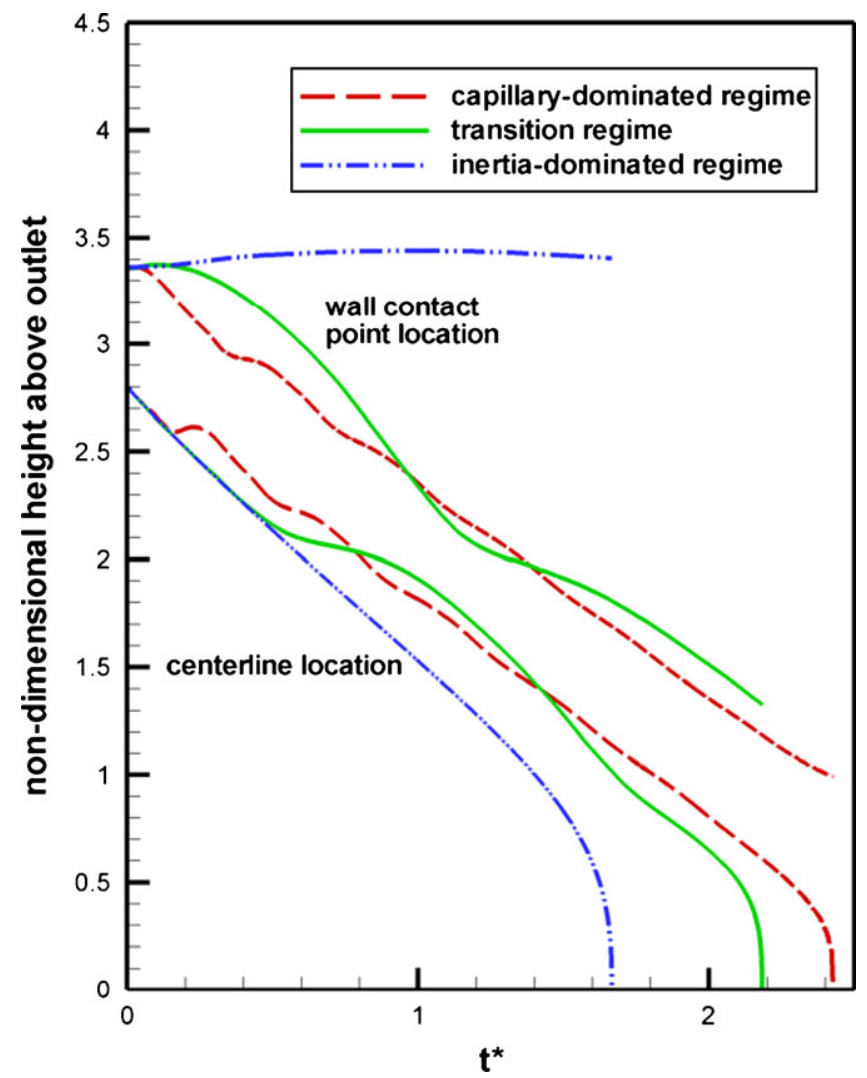

Figure 9. Non-dimensional height at the centerline and on the tank wall at capillarydominated $(W e /(B o+1)=0.03)$, transition $(W e /(B o+1)=0.3)$, and inertia-dominated regime $(W e /(B o+1)=13.3)$. Initial fill level is 3 tank radii.

with the same velocity both on the centerline and wall; simultaneously, it maintains its initial interface shape due to dominating capillary forces. The transition regime shows a few slosh waves with large amplitudes. The only sloshing wave observed in Figure 9 for the transition regime may not even be observed for smaller initial fill levels due to the short draining time. In the inertiadominated regime, the wall attachment point rarely moves; however, the interface at the centerline moves down with a constant velocity until vapor ingestion occurs. This regime is observed for large 


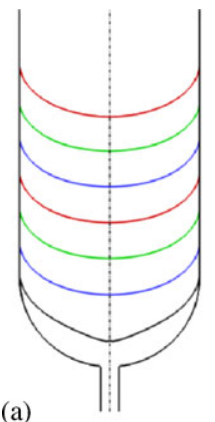

(a)

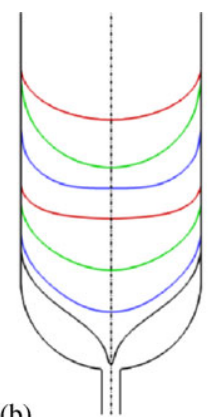

(b)

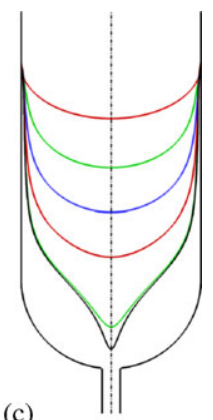

(c)

Figure 10. Snapshots of interface shape for each regime during draining process: (a) Capillary-dominated regime $(W e /(B o+1)=0.03)$; (b) transition regime $(W e /(B o+1)=0.33)$; and (c) inertia-dominated regime $(W e /(B o+1)=13.33)$.

draining parameters and causes a larger residual volume at the time of vapor ingestion because of the almost stationary wall attachment point.

Detailed interface shapes at different time steps are presented in Figure 10 for each regime. Figure 10(a) represents capillary-dominated regime for which the interface maintains its initial shape and moves with an almost-constant velocity until vapor ingestion occurs. The transition regime with large-amplitude slosh waves is shown in Figure 10(b). The interface heights at the centerline and at the wall attachment point move at different speeds when compared with the capillary-dominated regime. Consequently, the interface tends to vary between curving up and flattening out as illustrated in Figure 10(b). In an inertia-dominated regime, the draining happens significantly around the center of the tank, yielding a constant velocity at the centerline as a result of weak capillary forces being unable to pull up/down the other regions of the surface. As a result, an elongated interface shape with an almost fixed wall attachment point is observed as shown in Figure 10(c).

The non-dimensional period of slosh waves, $T^{*}$, is investigated for various draining parameters in Figure 11, which omits the inertia-dominated regime since waves do not exist for that regime. The wave period increases with the draining parameters from the capillary-dominated regime to the transition regime. In a capillary-dominated regime, waves have a small period and magnitude. The amplitude of waves becomes bigger as the draining parameter increases yielding large, noticeable waves with larger wave periods in the transition regime. As shown in Figure 11, such behavior is consistent with the single data point obtained by the experimental study of Symons [5]. Owing to experimental limitations of operating time under microgravity facility, no additional experimental data relevant to the wave period are available for further comparison. The present numerical study shows an almost linear correlation between the non-dimensional wave period and the draining parameter.

\subsection{Liquid fuel surface stability under thrust oscillation}

The influence of thrust oscillation on the liquid fuel tank is investigated. In a practical rocket system, thrust oscillation is composed of many different frequencies and magnitudes produced by different parts of the rocket [6]. The present study focused on the parametrically excited liquid fuel surface wave, namely the Faraday wave where the vertical sinusoidal oscillation in Equation (21) is applied to liquid/air in an open container, as depicted in Figure 12.

$$
a=g_{0}(1+\alpha \sin (2 \pi f t))
$$

where $g_{0}$ is standard gravity, $\alpha$ is oscillation magnitude ratio, and $f$ is forcing frequency. 


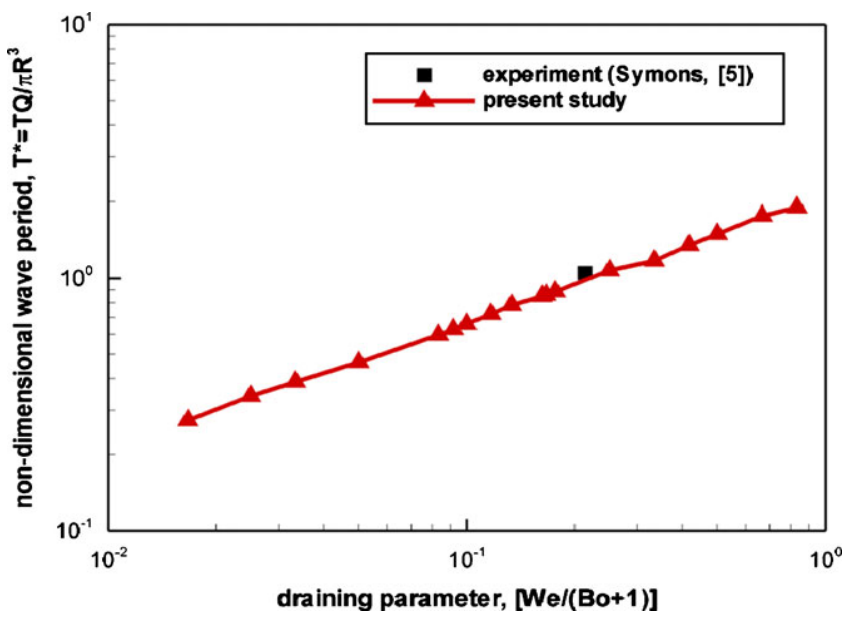

Figure 11. Non-dimensional wave period in a fuel tank with draining parameter.

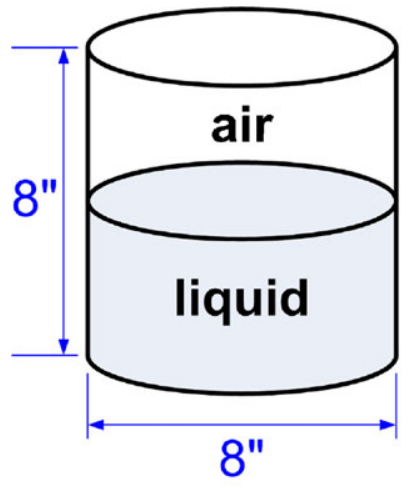

(a)

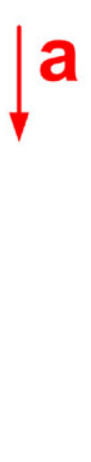

(b)

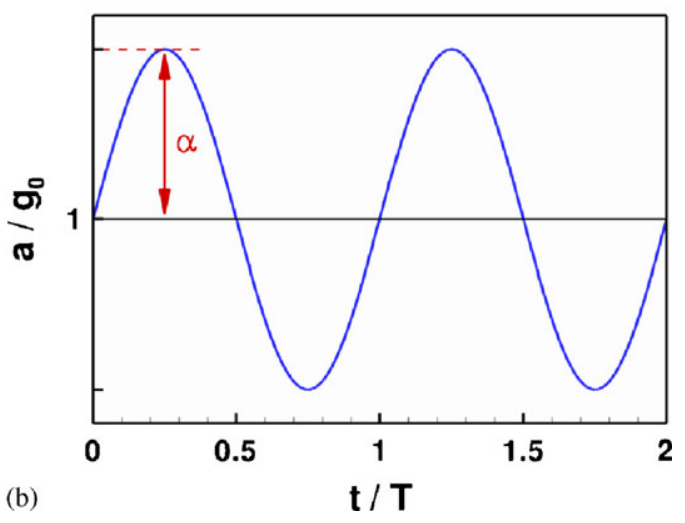

Figure 12. Configuration of parametrically excited Faraday waves: (a) 3-D geometric configuration of liquid/air in an open container and (b) vertical sinusoidal acceleration.

According to the dispersion relation for infinite depth periodic waves in Equation (22), the surface waves become unstable by restoring forces of either gravitational effects or surface tension effects [37].

$$
w^{2}=g k+\left(\frac{\sigma}{\rho}\right) k^{3}
$$

where $w$ is the angular frequency of the surface waves, $g$ is the local acceleration, and $k$ is the wave number. At a low wave number, the gravitational effect dominates; in addition, the surface tension effect dominates at a high wave number. The crossover wave frequency, $w_{\mathrm{c}}=\left(4 g^{3} \rho / \sigma\right)^{1 / 4}$, is determined from the crossover wave number, $k_{\mathrm{c}}=\sqrt{g \rho / \sigma}$, where the effects are equal. When the liquid fuel tank is parametrically excited with forcing angular frequency $w_{\mathrm{f}}=2 \pi f$, the energy is pumped into waves of angular frequency $w=w_{\mathrm{f}} / 2, w_{\mathrm{f}}, 3 w_{\mathrm{f}} / 2, \ldots$ It is known that the interface stability depends on the surface tension and kinematic viscosity. Goodridge et al. [14] defined the non-dimensionalized forcing frequency and forcing acceleration from the dimensional study in Equations (23) and (24).

$$
w^{*}=\frac{w_{0}}{(\sigma / \rho)^{2} / v^{3}}
$$



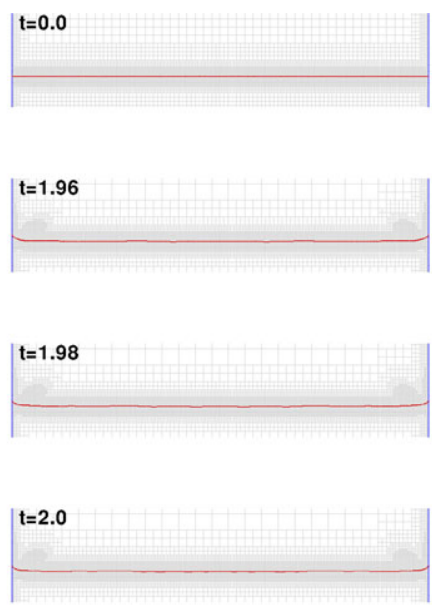

(a)
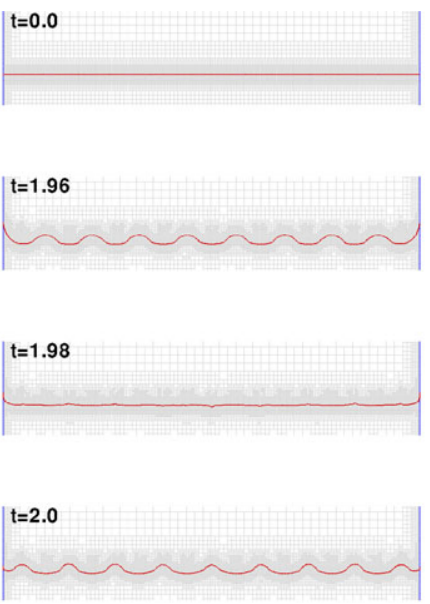

(b)
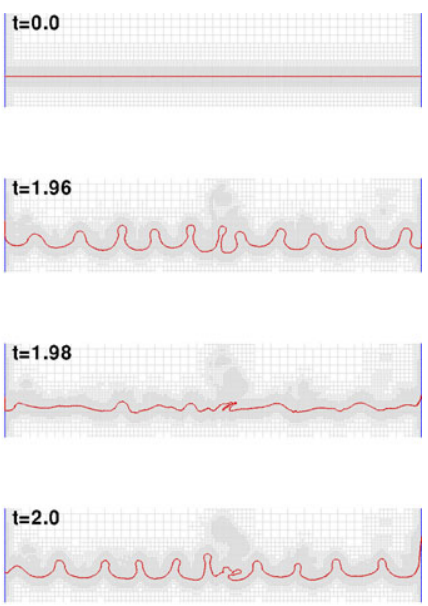

(c)

Figure 13. The features of surface wave stability as oscillation magnitude increases. Water/air fluids are vertically oscillated at a forcing frequency $f=20 \mathrm{~Hz}$. The flat liquid surface waves becomes periodic standing waves as oscillation magnitude increases, and shows the transition to unsteady aperiodic states with upward jets and droplets when the acceleration exceeds a certain threshold acceleration:

(a) $\alpha=0.1$; (b) $\alpha=0.5$; and (c) $\alpha=1.3$.

$$
a^{*}=\frac{a}{(\sigma / \rho)^{3} / v^{4}}
$$

Depicted in Figure 12, an open container with a depth of 8 in and a diameter (or width in 2-D) of 8 in is simulated to compare the present study with the experimental results conducted by Goodridge et al. [13-15]. Water and water-glycerin solutions with different viscosities are used to investigate the liquid surface stability. As is done in the experimental study, the contact angle between liquid and solid is assumed to be $0^{\circ}$. 2-D simulations are first conducted to check the overall tendency of stability due to their computational efficiency, and then 3-D cases are simulated for scaling analysis and comparison with the experiment.

Figure 13 shows the 2-D liquid surface wave evolution in time for water/air fluids as oscillation magnitude increases at a given forcing frequency $f=20 \mathrm{~Hz}$. With low acceleration, it shows a flat surface wave in Figure 13(a), but it becomes a periodic standing wave in Figure 13(b) as forcing acceleration increases. When the acceleration exceeds a certain threshold level, referred to threshold acceleration, the stable periodic surface waves are transformed to an unstable aperiodic state and show some upward jets in Figure 13(c). Goodridge et al. [14] defined the threshold acceleration as the acceleration level where two droplets are detected within 10s. Das and Hopfinger [10], however, defined it as a jet forming or wave crest pinching off, followed by irregular motion. In this study, droplets or long filament-like jet formations are assumed as criteria for threshold acceleration because droplet formation itself in numerical studies may result in a different criterion according to grid resolution.

The influence of a fluid's viscosity on the threshold acceleration is investigated. Both water and $80 \%$ glycerin-water solution, which has a kinematic viscosity $(v) 50$ times larger than water's, are considered. A higher kinematic viscosity makes the liquid surface waves more stable. This results in a higher threshold acceleration before aperiodic wave states appear with upward jets. Another feature of higher viscosity is that it creates longer filament-like jets than lower viscosity fluid, as is shown in Figure 14.

According to the dispersion relation for infinite depth periodic waves in Equation (22), as the forcing frequency increases, the gravity effect is less important, and the capillary effect becomes more dominant. Figure 15(a) shows gravity waves at forcing frequency $f=10 \mathrm{~Hz}$, which is lower than the crossover forcing frequency $\left(f_{\mathrm{c}}=2 w_{\mathrm{c}} /(2 \pi)=27 \mathrm{~Hz}\right.$ in water/air case). It shows unsteady aperiodic states with a very large upward jet on the centerline of the container. The transition from 
(a)
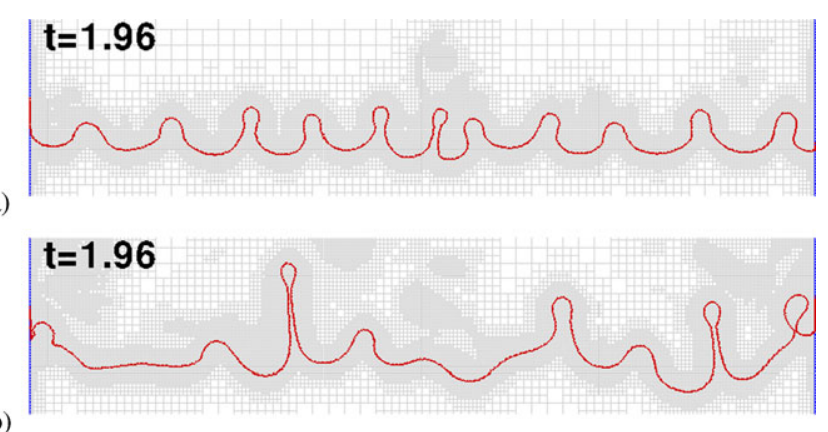

Figure 14. The effect of fluid viscosity at forcing frequency $f=20 \mathrm{~Hz}$. Higher viscosity of fluid shows longer filament-like jets with higher threshold acceleration: (a) Water, $\alpha=1.3$ and (b) $80 \%$ glycerin-water $\left(v=50 \times v_{\text {water }}\right), \alpha=2$.

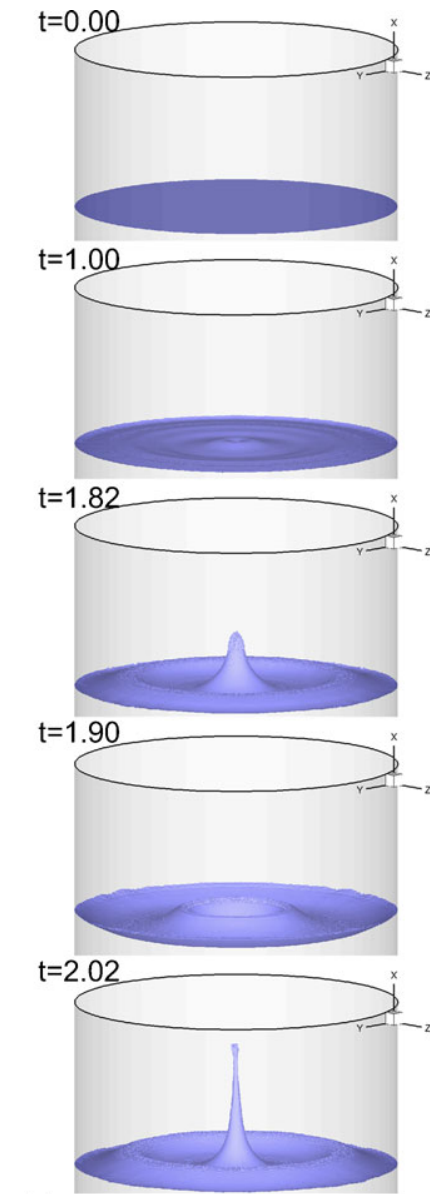

(a)

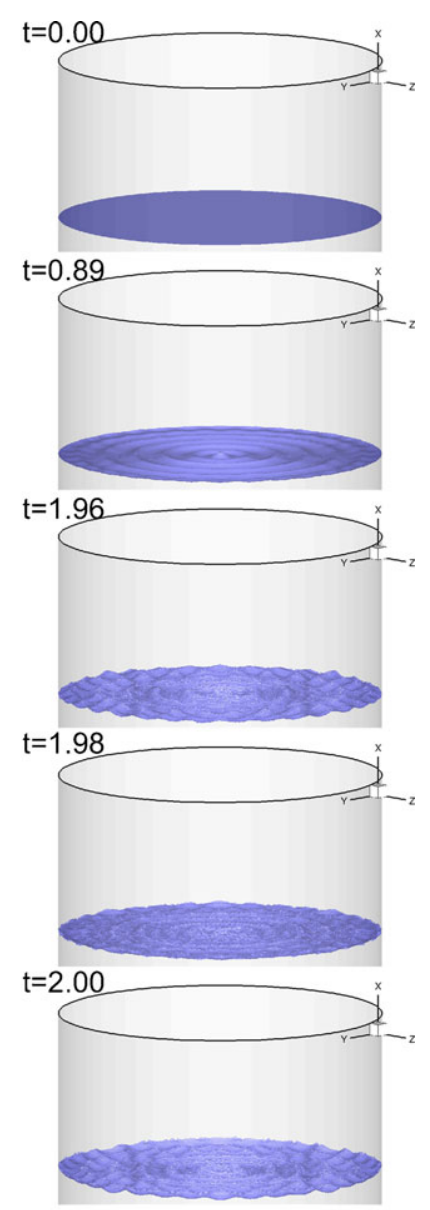

(b)

Figure 15. Comparison between gravity waves and capillary-transition waves in aperiodic surface states for water/air case: (a) $f=10 \mathrm{~Hz}$ and $\alpha=0.3$ and (b) $f=20 \mathrm{~Hz}$ and $\alpha=0.6$.

the gravity wave into the capillary wave is observed in Figure 15(b), where the large upward jet in the center of the axis does not exist, and smaller multiple jets are shown in multiple locations. It is noted that a higher forcing frequency results in a smaller wave length, and consequently smaller multiple jets. The grids are adapted dynamically up to 200 grid points along the tank diameter, in order to capture the complex surface wave motions. We can find that 3-D computation capability 


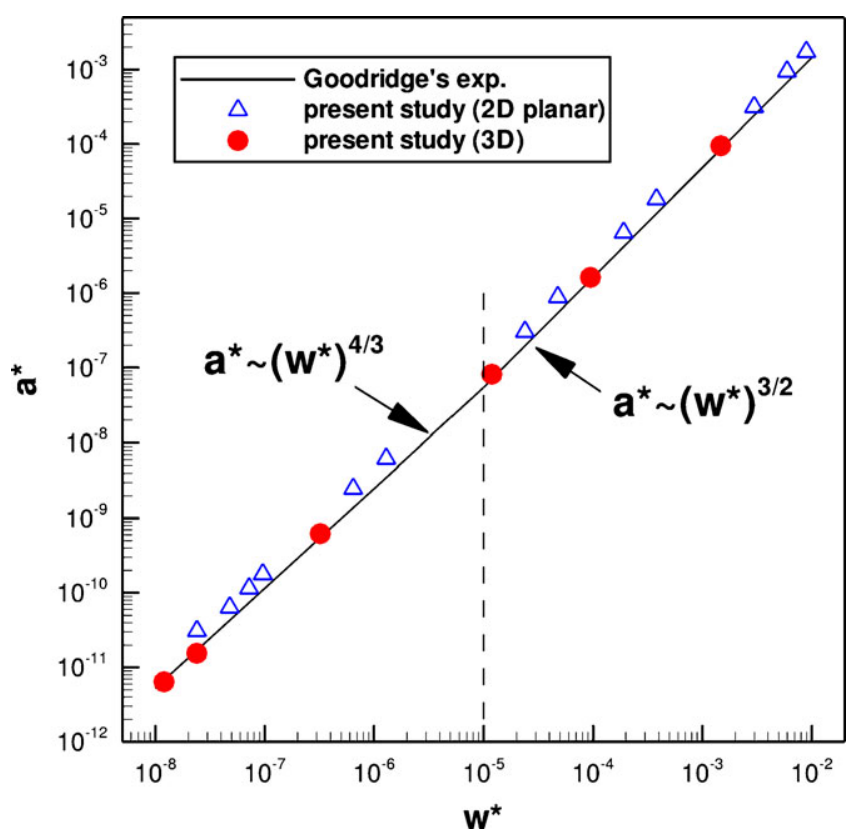

Figure 16. Non-dimensionalized threshold acceleration as a function of non-dimensionalized angular forcing frequency. Lower-viscosity region $\left(w^{*}<10^{-5}\right)$ and higher-viscosity region $\left(w^{*}>10^{-5}\right)$ show different correlations between threshold acceleration and forcing frequency.

with a fine grid is required to capture the capillary wave motions with multiple jets as shown in Figure 15(b).

The non-dimensional threshold acceleration $\left(a^{*}=a v^{4} /(\sigma / \rho)^{3}\right)$ is plotted with non-dimensional angular forcing frequency $\left(w^{*}=w_{\mathrm{f}} v^{3} /(\sigma / \rho)^{2}\right)$ in Figure 16. Both 2-D and 3-D computations are in good agreement with Goodridge's experiment. However the 2-D simulation shows slightly higher threshold acceleration than the 3-D simulation. The 2-D planar case requires more forcing energy to form upward jets since it needs to push up more mass than the 3-D. These result in higher threshold acceleration in 2-D. However, the overall trend matches very well. The nondimensionalized threshold acceleration shows $\left(w^{*}\right)^{4 / 3}$ dependence for the lower-viscosity region $\left(w^{*}<10^{-5}\right)$, and $\left(w^{*}\right)^{3 / 2}$ dependence for the higher-viscosity region $\left(w^{*}>10^{-5}\right)$. This indicates that each region is dominated by different parameters. The correlation study on the dimensional parameters shows it clearly. For the low-viscosity region, the threshold acceleration can be correlated by $a \sim(\sigma / \rho)^{1 / 3} w_{0}^{4 / 3}$ correlation, and thus the flow is dominated by surface tension and forcing frequency. For the high-viscosity flow region, the viscosity itself and the forcing frequency dominate the surface stability by $a \sim v^{1 / 2} w_{0}^{3 / 2}$ dependence.

\section{CONCLUSION}

In this paper, a three-dimensional adaptive Eulerian-Lagrangian method is developed to study the interfacial dynamics of multiphase flow in a spacecraft environment. The governing equations are solved on an Eulerian Cartesian grid with dynamic local grid adaptation for appropriate resolution. The Lagrangian-triangulated surface meshes are implemented for tracking interfaces including different fluid phase boundaries and arbitrarily shaped solid geometries. Both continuous and sharp interface methods are implemented in a unified framework using the contact line force model with the local slip condition. Special attention is given to offer satisfactory treatment of moving fluid-fluid boundaries based on the continuous interface treatment, as well as solid boundary based on the sharp interface method with the ghost fluid concept. 
Two fluid flow problems related to the draining and stability of liquid fuel in a spacecraft fuel tank are numerically performed, and the results agree well with the experimental observations. Specifically, the following are observed:

(i) Liquid fuel draining dynamics under microgravity.

- Three different flow regimes are observed in the draining process under microgravity condition: a capillary-dominated regime, a transition regime, and an inertia-dominated regime.

- The liquid residual increases with oscillation as the draining parameter increases, and it remains almost constant at a large draining parameter.

- The non-dimensional slosh wave period increases with the draining parameter.

(ii) Liquid fuel surface stability under vertical sinusoidal oscillation of acceleration.

- As the forcing oscillation magnitude increases, the surface wave exhibits periodic standing waves, and reaches aperiodic unstable states with upward jets and droplets when the forcing magnitude exceeds a certain threshold value, namely the threshold acceleration.

- The threshold acceleration increases with the forcing frequency.

- As the kinematic viscosity of a fluid increases, the threshold acceleration increases with longer filament-like upward jets.

- As expected from the dispersion relation for infinite depth periodic wave, the gravity wave is observed when the forcing frequency is lower than the crossover wave frequency. The capillary-transition wave is observed as the forcing frequency increases.

- For lower viscosity fluids, the surface tension dominates the surface stability, and $a^{*} \sim$ $\left(w^{*}\right)^{4 / 3}$ scaling is observed from both Goodridge's experiment and the present simulations.

- For higher viscosity fluids, the viscosity becomes an important parameter, and $a^{*} \sim\left(w^{*}\right)^{3 / 2}$ scaling is observed from both Goodridge's experiment and the present simulations.

\section{ACKNOWLEDGEMENTS}

The work reported in this paper has been partially supported by NASA Constellation University Institutes Program (CUIP), Claudia Meyer and Jeff Rybak program managers. We are benefitted from the discussion with John Peugeot and Jeff West of NASA Marshall Space Flight Center.

\section{REFERENCES}

1. Petrash DA, Zappa RF, Otto EW. Experimental study of the effects of weightlessness on the configuration of mercury and alcohol in spherical tanks. NASA TN D-1197, 1962.

2. Nussle RC, Derdul JD, Petrash DA. Photographic study of propellant outflow from a cylindrical tank during weightlessness. NASA TN D-2572, 1965.

3. Derdul JD, Grubb LS, Petrash DA. Experimental investigation of liquid outflow from cylindrical tanks during weightlessness. NASA TN D-3746, 1966.

4. Berenyi SG, Abdalla KL. Vapor ingestion phenomenon in hemispherically bottomed tanks in normal gravity and in weightlessness. NASA TN D-5704, 1970.

5. Symons EP. Draining characteristics of hemispherically bottomed cylinders in a low-gravity environment. NASA TP-1297, 1978.

6. Yang V, Andersen WE. Liquid Rocket Engine Combustion Instability. AIAA: New York, 1995.

7. Faraday M. On a peculiar class of acoustical figures; and on certain forms assumed by groups of particles upon vibrating elastic surfaces. Philosophical Transactions of the Royal Society of London 1831; 121:299-340.

8. Simonelli F, Gollub JP. Surface wave mode interactions: effects of symmetry and degeneracy. Journal of Fluid Mechanics 1989; 199:471-494.

9. Miles J, Henderson D. Parametrically forced surface waves. Annual Review of Fluid Mechanics 1990; 22:143-165.

10. Das SP, Hopfinger EJ. Parametrically forced gravity waves in a circular cylinder and finite-time singularity. Journal of Fluid Mechanics 2008; 599:205-228.

11. Perinet N, Juric D, Tuckerman L. Numerical simulation of Faraday waves. Journal of Fluid Mechanics 2009; 635: $1-26$.

12. Perlin M, Schultz WW. Capillary effects on surface waves. Annual Review of Fluid Mechanics 2000; 32:241-274.

13. Goodridge CL, Shi WT, Lathrop DP. Threshold dynamics of singular gravity-capillary waves. Physical Review Letters 1996; 77(22):4692. 
14. Goodridge CL, Shi WT, Hentschel HGE, Lathrop DP. Viscous effects in droplet-ejecting capillary waves. Physical Review E 1997; 56(1):472.

15. Goodridge CL, Hentschel HGE, Lathrop DP. Breaking Faraday waves: critical slowing of droplet ejection rates. Physical Review Letters 1999; 82(15):3062.

16. Shyy W, Udaykumar HS, Rao MM, Smith RW. Computational Fluid Dynamics with Moving Boundaries. Taylor \& Francis: Philadelphia, 1996.

17. Prosperetti A, Tryggvason G. Computational Methods for Multiphase Flow. Cambridge University Press: New York, 2007.

18. Ryskin G, Leal LG. Numerical solution of free-boundary problems in fluid mechanics. Part 2. Buoyancy-driven motion of a gas bubble through a quiescent liquid. Journal of Fluid Mechanics 1984; 148:19-35.

19. Perot B, Nallapati R. A moving unstructured staggered mesh method for the simulation of incompressible free-surface flows. Journal of Computational Physics 2003; 184(1):192-214.

20. Osher S, Fedkiw R. Level Set Methods and Dynamic Implicit Surfaces. Springer: New York, 2002.

21. Hirt CW, Nichols BD. Volume of fluid (VOF) method for the dynamics of free boundaries. Journal of Computational Physics 1981; 39(1):201-225.

22. Peskin CS. The immersed boundary method. Acta Numerica 2003; 11:479-517.

23. Francois M, Shyy W. Micro-scale drop dynamics for heat transfer enhancement. Progress in Aerospace Sciences 2002; 38(4):275-304.

24. Francois M, Shyy W. Computations of drop dynamics with the immersed boundary method, part 1: numerical algorithm and buoyancy-induced effect. Numerical Heat Transfer: Part B: Fundamentals 2003; 44(2):101-118.

25. Francois M, Shyy W. Computations of drop dynamics with the immersed boundary method, part 2: drop impact and heat transfer. Numerical Heat Transfer: Part B: Fundamentals 2003; 44(2):119-143.

26. Udaykumar HS, Mittal R, Rampunggoon P, Khanna A. A sharp interface cartesian grid method for simulating flows with complex moving boundaries. Journal of Computational Physics 2001; 174(1):345-380.

27. Ye T, Mittal R, Udaykumar HS, Shyy W. An accurate Cartesian grid method for viscous incompressible flows with complex immersed boundaries. Journal of Computational Physics 1999; 156(2):209-240.

28. Ye T, Shyy W, Chung JN. A fixed-grid, sharp-interface method for bubble dynamics and phase change. Journal of Computational Physics 2001; 174(2):781-815.

29. Tryggvason G, Bunner B, Esmaeeli A, Al-Rawahi N, Tauber W, Han J, Jan YJ, Juric D, Nas S. A front-tracking method for the computations of multiphase flow. Journal of Computational Physics 2001; 169(2):708-759.

30. Singh R, Shyy W. Three-dimensional adaptive Cartesian grid method with conservative interface restructuring and reconstruction. Journal of Computational Physics 2007; 224(1):150-167.

31. Ye T, Shyy W, Chung JN. A fixed-grid, sharp-interface method for bubble dynamics and phase change. Journal of Computational Physics 2001; 174(2):781-815.

32. Mittal R, Dong H, Bozkurttas M, Najjar FM, Vargas A, von Loebbecke A. A versatile sharp interface immersed boundary method for incompressible flows with complex boundaries. Journal of Computational Physics 2008; 227(10):4825-4852.

33. Udaykumar HS, Shyy W, Rao MM. Elafint: a mixed Eulerian-Lagrangian method for fluid flows with complex moving boundaries. International Journal for Numerical Methods in Fluids 1996; 22(8):691-712.

34. Liu XD, Fedkiw RP, Kang M. A boundary condition capturing method for Poisson's equation on irregular domains. Journal of Computational Physics 2000; 160(1):151-178.

35. Uzgoren E, Sim J, Shyy W. Marker-based, 3-D adaptive Cartesian grid method for multiphase flow around irregular geometries. Communications in Computational Physics 2009; 5(1):1-41.

36. Sim J, Shyy W. 3-D multiscale adaptive Eulerian-Lagrangian method for multiphase flows with phase change. Forty-eighth AIAA Aerospace Science Meeting, AIAA 2010-1295, 2010.

37. Landau LD, Lifshitz EM. Fluid Mechanics. Pergamon Press: New York, 1987. 